\title{
Evaluación de la sobrecarga en los cuidadores de los pacientes con enfermedad de Parkinson ambulatorios y sus factores de riesgo
}

\author{
OLGA BENAVIDES, DANIELA ALBURQUERQUE, PEDRO CHANÁ-CUEVAS
}

Centro de Trastornos del Movimiento (CETRAM), Facultad de Ciencias Médicas, Universidad de Santiago de Chile, Santiago, Chile.

Recibido el 17 de agosto de 2012, Aceptado el 6 de noviembre de 2012

Correspondenc a: Pedro Chaná-Cuevas Centro de Trastornos del Movimiento (CETRAM), Universidad de Santiago de Chile.

Belisario Prats 1597 B Independencia Santiago, Chile. E-mail: pedro.chana@ usach.cl

\section{Burden among caregivers of patients with Parkinson disease}

Background: Parkinson disease (EPI) patients often require being assisted by others. These caregivers are exposed to a decrease in their quality of life. Aim: To explore Parkinson disease patient features associated with a greater burden among their caregivers. Material and Methods: Fifty one patients with Parkinson disease (aged $67 \pm 12$ years, 29 men, with $8 \pm 5$ years of disease) and their caregivers, were studied. Patients were assessed with the Unified Parkinson Disease Rating Scale III, the Hoehn \& Yahr stage standardization, Parkinson's minimental test, the neuropsychiatric inventory and the Beck Depression Inventory (IDB). The Zarit Burden Interview (ESZ) was applied to caregivers. Results: According to IDB, 45\% of patients whose caregivers presented little or no burden had a depression, compared to $78 \%$ of those whose caregivers had moderate or intense burden. $(p<0.01)$. The ESZ score of caregivers correlated significantly with Parkinson patients' age, IDB and axial involvement in the UPDRS-III (correlation coefficients of $0.4 p<0.01,0.6 p<0.01$ and $0.46 p<0.01$, respectively). Conclusions: Motor alterations, cognitive impairment and most importantly depression of patients with Parkinson disease are deteminants of burden for their caregivers.

(Rev Med Chile 2013; 141: 320-326).

Key words: Caregivers; Depression; Parkinson Disease; Quality of Life.
L a enfermedad de Parkinson idiopática (EPI) es un cuadro neurológico, crónico progresivo e irrecuperable que afecta a aproximadamente el 1,9 por mil de la población. Su prevalencia aumenta en los mayores de 60 años a 1\%, siendo un problema relevante de salud pública ${ }^{1,2}$. Tradicionalmente, los estudios se han focalizado en los aspectos motores de la enfermedad. En estos últimos tiempos, con los avances terapéuticos en esta área, se han tornado relevantes también otros aspectos como la depresión, deterioro cognitivo, los trastornos conductuales, entre otros, los que se han denominado aspectos no motores ${ }^{3,4}$.

Sin embargo, el impacto de los diferentes aspectos de la enfermedad sobre los cuidadores es un tema poco abordado. Los estudios existentes se han focalizado preferentemente en la demencia y su efecto sobre el cuidador ${ }^{5-8}$. Estudiamos una población de pacientes EPI ambulatoria que consulta en nuestro centro, con el objeto de evaluar los factores del paciente que inciden en la sobrecarga del cuidador y los factores de riesgo de estos últimos.

\section{Material y Métodos}

El presente estudio es de carácter descriptivo y correlacional. La muestra se reunió entre marzo y abril de 2011 en pacientes ambulatorios que consultan en el centro de trastornos del movimiento. 
Sobrecarga de cuidador en la enfermedad de Parkinson - O. Benavides et al

Los criterios de inclusión para los pacientes EPI fueron cumplir los criterios establecidos por el Banco de Cerebros de Londres ${ }^{9}$, reconocer a un cuidador principal y aceptar voluntariamente participar en el estudio. Se excluyeron del estudio los pacientes con otros diagnósticos neurológicos. En el caso de los cuidadores, los criterios fueron ser reconocido como cuidador principal por el paciente y aceptar voluntariamente participar en el estudio.

Para los fines de la investigación, las variables se definieron de la siguiente manera:

- Carga del cuidador: sentimientos negativos y experiencia tensional como resultado de cuidar a una persona con enfermedad crónica. Evaluado mediante la escala de sobrecarga del cuidador de Zarit ${ }^{9}$.

- Cuidador principal: persona de la familia o cercana al paciente que se ocupa de darle apoyo físico y/o emocional en forma permanente y comprometida ${ }^{10}$.

Después de aplicar los criterios de inclusión al total de 59 pacientes, la muestra quedó constituida por 51 pacientes y sus cuidadores. Ocho pacientes fueron excluidos porque no reconocieron un cuidador principal. Las características demográficas de pacientes y cuidadores se resumen en la Tabla 1. Las variables estudiadas en los pacientes fueron datos demográficos y tiempo de evolución de la enfermedad. El tratamiento anti-parkinsoniano fue expresado en equivalente de levodopa ${ }^{11}$.

Los sujetos fueron sometidos a una evaluación clínica neurológica consistente en historia clínica, examen físico y neurológico y se administró la Escala unificada para la evaluación de la enfermedad de Parkinson en su sección motora III (UPDRS-III) y la etapificación de Hoehn \& Yahr ${ }^{12}$. La UPDRS-III fue analizada en su puntaje total y se establecieron los siguientes subíndices: Temblor ítem 20-21-22, Rigidez ítem 24-25, Bradicinesia ítem 26 a 29 y compromiso axial ítem 30-31-3233 y $34^{13}$. En la evaluación neuropsicológica se les administró a todos los pacientes el Test Minimental Parkinson (MMP) que fue analizado en su puntaje total. El punto de corte para este test es de 24 puntos en la detección de demencia ${ }^{14}$.

Se administró el inventario neuropsiquiátrico (NPI), que fue evaluado en su puntaje total y su frecuencia en los diferentes ítems ${ }^{15,16}$. El Inventario de Beck (IBD) aplicado para depresión versión auto-administrada, que se evalúa en 13 ítems, considerándose los siguientes puntos de corte: de 0 a 4 depresión ausente o mínima; 5 a 7 depresión leve; 8 a 15 depresión moderada y mayor de 15 depresión grave ${ }^{17}$. Todas las evoluciones se realizaron durante la misma sesión y en estado "ON" del paciente.

Respecto de los cuidadores, se les solicitaron datos demográficos que se resumen en la Tabla 1, además del tiempo que compartían con el paciente en porcentaje del día. Asimismo, se les solicitó información sobre su nivel educacional y completar la escala de sobrecarga para el cuidador de Zarit, versión auto-administrada $(E S Z)^{18}$. Ésta explora los efectos de impacto sobre el aumento de los índices de sobrecarga del cuidador. Su puntaje global va de 22 a 110 y se clasifican entre 22-46 puntos sin sobrecarga; $47-55$ con sobrecarga leve; y 56-110 sobrecarga intensa. Se distinguen tres componentes en el test impacto: interpersonal,

\section{Tabla 1. Características de la población de pacientes con enfermedad de Parkinson y los cuidadores}

\begin{tabular}{|ll|}
\hline Pacientes & \\
\hline $\begin{array}{l}\text { Sexo Masculino/Femenino } \\
\text { Estadios de Hoehn \& Yahr }\end{array}$ & $29(56,8 \%) / 22(43,1 \%)$ \\
$\quad$ II & $4(7,8 \%)$ \\
$\quad$ IV & $22(43,2 \%)$ \\
\hline $\begin{array}{l}\text { Pacientes que usan levodopa } \\
\text { Pacientes que usan agonistas }\end{array}$ & $49(96,1 \%)$ \\
dopaminérgicos & $13(25,5 \%)$ \\
\hline $\begin{array}{l}\text { Edad al examen, años } \\
\text { Edad al inicio de la enfermedad, } \\
\text { años }\end{array}$ & $67,5 \pm 12,8$ \\
\hline $\begin{array}{l}\text { Duración de la enfermedad, } \\
\text { años }\end{array}$ & $58,7 \pm 13,5$ \\
\hline $\begin{array}{l}\text { Total UPDRS sección III (total } \\
\text { rango 0-108) }\end{array}$ & $32,1 \pm 18,3$ \\
\hline $\begin{array}{l}\text { Dosis de Levodopa día, mg } \\
\text { Cuidadores }\end{array}$ & $830 \pm 434$ \\
\hline $\begin{array}{l}\text { Sexo Masculino/Femenino } \\
\text { Relación (esposa-o/hija-o) }\end{array}$ & $18(35,3) / 33(64,7)$ \\
\hline $\begin{array}{l}\text { Porcentaje del día que está con } \\
\text { el paciente }\end{array}$ & $91,1 \pm 22,6$ \\
\hline
\end{tabular}


competencia y expectativas ${ }^{19}$. Esto en la versión validada para población adulto mayor chilena ${ }^{20}$.

Los resultados se expresaron en medias \pm desviaciones estándar. Las comparaciones se realizaron con $\chi^{2}$ y la t de Student y las correlaciones $\mathrm{R}$ de Sperman y de Pearson. Se utilizó el paquete estadístico SPSS versión 15 para Windows. Se consideró estadísticamente significativo una $\mathrm{p}<0,05$.

\section{Resultados}

Al ser separados los 51 pacientes con enfermedad de Parkinson evaluados (29 hombres (56,8\%) y 22 mujeres $(43,1 \%)$ los resultados fueron los siguientes: en un grupo de 33 pacientes (19 hombres y 14 mujeres), los cuidadores presentaron un puntaje menor a 55 en la ESZ, clasificados como "sin sobrecarga o sobrecarga leve". En otro grupo de 18 pacientes ( 10 hombres y 8 mujeres), los cuidadores tenían un puntaje mayor o igual a 55 en la ESZ, clasificados con "sobrecarga intensa". En la Tabla 2 se presentan las características de los pacientes y sus cuidadores principales, respectivamente.

En nuestra muestra todos los cuidadores eran familiares. En 39 casos (76,5\%) el esposo/a y en 12 casos $(23,5 \%)$ hijo/a. La escolaridad de los cuidadores resultó de 15 casos con enseñanza básica, 21 con enseñanza media y 11 con enseñanza superior. La ESZ en relación al parentesco, demostró para las esposas o esposos un puntaje promedio de 48,0 $\pm 17,5$ y para los hijos o hijas un puntaje promedio de 50,9 $\pm 15,7$, diferencia no significativa estadísticamente. Los esposos/as comparten 94,6 \pm $16,9 \%$ de su tiempo con el paciente, en tanto que los hijos 79,1 $\pm 35,8 \%$, diferencia no significativa estadísticamente.

Se analizaron también los factores de riesgo de la enfermedad de Parkinson en relación a la sobrecarga del cuidador presentada en la Tabla 3.

\section{Trastornos motores (UPDRS, H\&Y, S\&E)}

Las fluctuaciones motoras estaban presentes en los pacientes donde el cuidador no presentaba sobrecarga, o si la presentaba era leve, 45,5\% (15 de 33 casos). Para los cuidadores con sobrecarga intensa de 77,8\% (14 de 18 casos), la diferencia fue analizada con $\chi^{2}$ demostrando un resultado estadísticamente significativo ( $\mathrm{p}<0,01)$.

La función motora se analizó mediante subíndices previamente definidos no encontrando diferencia estadísticamente significativa para los índices de temblor, rigidez, ni bradicinesia. El índice de compromiso axial en los pacientes donde el cuidador no presentaba sobrecarga o era leve fue de 7,3 $\pm 4,4$ y para los con sobrecarga intensa de $12,5 \pm 7,5(\mathrm{p}<0,01)$.

\section{Trastornos cognitivos (MMP)}

Se realizó MMP en 39 casos. Los resultados con 24 puntos o menos fueron aquellos en los que el

Tabla 2. Característica demográfica de los pacientes y cuidadores en relación a la sobrecarga del cuidador

\begin{tabular}{|c|c|c|c|}
\hline & \multicolumn{2}{|c|}{ Cuidador } & \multirow[t]{2}{*}{ t de Studens } \\
\hline & $\begin{array}{l}\text { Sin sobrecarga o } \\
\text { sobrecarga leve }\end{array}$ & $\begin{array}{c}\text { Sobrecarga } \\
\text { intensa }\end{array}$ & \\
\hline \multicolumn{4}{|l|}{ Paciente } \\
\hline Número (masculino/femenino) & $33(19 / 14)$ & $18(10 / 8)$ & \\
\hline Edad al momento del examen & $65,0 \pm 14,3$ & $72,0 \pm 7,9$ & $p<0,05$ \\
\hline Edad de inicio de la enfermedad & $57,8 \pm 15,1$ & $61,9 \pm 11,5$ & \\
\hline Tiempo de evolución de la enfermedad & $7,2 \pm 4,9$ & $10,1 \pm 5,9$ & \\
\hline Equivalentes de levodopa total & $717 \pm 384$ & $1.038 \pm 454$ & $p<0,05$ \\
\hline \multicolumn{4}{|l|}{ Cuidadores } \\
\hline Cuidadores sexo (masculino/femenino) & $32(13 / 19)$ & $19(5 / 14)$ & \\
\hline Edad del cuidador & $61,9 \pm 15,6$ & $63,6 \pm 11,3$ & \\
\hline Dedicación del cuidador al paciente en \% del día & $86,0 \pm 27,9$ & $98,9 \pm 4,6$ & $p<0,05$ \\
\hline Relación con el paciente (esposa/hijo) & $26 / 6$ & $13 / 6$ & \\
\hline Escala de sobrecarga del cuidador de Zarit & $38,7 \pm 9,6$ & $66,9 \pm 12,4$ & $p<0,001$ \\
\hline
\end{tabular}


Sobrecarga de cuidador en la enfermedad de Parkinson - O. Benavides et al

Tabla 3. Factores de riesgo de la enfermedad de Parkinson en relación a la sobrecarga del cuidador

\begin{tabular}{|lcc|}
\hline & \multicolumn{2}{c|}{$\begin{array}{c}\text { Cuidador } \\
\text { Studens }\end{array}$} \\
Número casos & $\begin{array}{c}\text { Sin sobrecarga o } \\
\text { sobrecarga leve }\end{array}$ & $\begin{array}{c}\text { Sobrecarga } \\
\text { intensa }\end{array}$ \\
UPDRS-III & 32 & 19 \\
\hline Etapificación de Hoehn \& Yahr & $29,6 \pm 14,5$ & $36,2 \pm 23,2$ \\
\hline Escala de actividades de la vida diaria de Schwab \& England & $2,5 \pm 0,7$ & $2,8 \pm 0,9$ \\
\hline Mini mental Parkinson & $66,6 \pm 18,1$ & $56,8 \pm 18,6$ \\
Inventario neuropsiquiátrico & $26,1 \pm 6,2$ & $23,6 \pm 4,3$ \\
\hline Inventario de Beck para depresión & $13,4 \pm 12,1$ & $19,1 \pm 10,9$ \\
\hline
\end{tabular}

Tabla 4. Correlaciones entre escala de sobrecarga de Zarit sus componentes y los factores de riesgo del paciente

\begin{tabular}{|lcccc|}
\hline & Factor impacto & $\begin{array}{c}\text { Factor } \\
\text { interpersonal }\end{array}$ & $\begin{array}{c}\text { Factor competencia } \\
\text { y expectativas }\end{array}$ & Escala global \\
\hline Edad & $R=0,315$ & $\mathrm{R}=0,287$ & $\mathrm{R}=0,565$ & $\mathrm{R}=0,4$ \\
& $\mathrm{p}<0,05$ & $\mathrm{p}<0,05$ & $\mathrm{p}<0,05$ & $\mathrm{p}<0,005$ \\
Inventario depresión de Beck & $\mathrm{R}=0,631$ & $\mathrm{R}=0,460$ & $\mathrm{NS}$ & $\mathrm{R}=0,615$ \\
& $\mathrm{p}<0,001$ & $\mathrm{p}<0,005$ & $\mathrm{~N}<0,001$ \\
Mini Mental Parkinson & $\mathrm{NS}$ & $\mathrm{R}=-0,325$ & $\mathrm{NS}$ & $\mathrm{NS}$ \\
Índice compromiso axial UPDRS & $\mathrm{R}=0,481$ & $\mathrm{R}=0,353$ & $\mathrm{NS}$ & $\mathrm{R}=0,461$ \\
& $\mathrm{p}<0,005$ & $\mathrm{p}<0,05$ & $\mathrm{p}<0,005$ \\
\hline
\end{tabular}

Correlaciones de Pearson.

cuidador no presentaba sobrecarga o ésta era leve, de $25,0 \%$ ( 6 de 24 casos). En los casos con sobrecarga intensa, de $60 \%$ ( 9 de 15 casos), la diferencia fue analizada con $\chi^{2}$, donde fue estadísticamente significativa $(\mathrm{p}<0,05)$.

\section{Trastornos conductuales (NPI)}

El porcentaje de casos con delirio y alucinaciones se presentó en los pacientes donde el cuidador no presentaba sobrecarga o es leve, de 18,2\% (6 de 33 casos). Para los con sobrecarga intensa, de $22,2 \%$ ( 4 de 18 casos) las diferencia observadas no alcanzan significación estadística.

\section{Trastornos anímicos (IBD)}

En relación al IBD, dio como resultado depresión moderada a severa en los pacientes donde el cuidador no presentaba sobrecarga o era leve, de $45,5 \%$ ( 15 de 33 casos), de los cuales 4 casos clasificaban en el rango de severas. Para los con sobre- carga intensa, de 77,8\% (14 de 18 casos), 9 casos clasificaban en el rango de severas. Esta diferencia fue analizada con $\chi^{2}$ resultando estadísticamente significativa $(\mathrm{p}<0,01)$. En la Tabla 3 se presentan las medias del puntaje total de la escala de IBD.

Escala de sobrecarga de Zarit: (ESZ)

$\mathrm{Al}$ correlacionar los diferentes componentes de la ESZ con los factores de riesgo evaluado en la Tabla 4 se resumen las correlaciones estadísticamente significativas encontradas.

\section{Discusión}

\section{Características de la población estudiada en relación a la sobrecarga}

Para el análisis se distinguieron dos grupos de pacientes: por un lado, aquellos en que el cuidador no presentaba sobrecarga o esta era leve al ser 
evaluados con la ESZ, y por otro lado, aquellos que tenían una sobrecarga intensa. La edad de los pacientes se correlaciona con una mayor sobrecarga al cuidador. Este factor se relaciona con un mayor compromiso axial, de la marcha, aspecto motor de la EPI y con la presencia de deterioro cognitivo ${ }^{21}$. Probablemente sean estos los elementos que determinan una mayor sobrecarga del cuidador por limitar la independencia del paciente.

\section{Característica de los cuidadores}

En nuestra muestra, los cuidadores fueron en todos los casos parientes, no existiendo cuidadores profesionales. Lo anterior probablemente se debe al factor económico propio de nuestra población con menor accesibilidad a este tipo de servicios ${ }^{22}$. En otras series, como la de Aarsland et $\mathrm{al}^{5}$ con énfasis en los trastorno neuropsicológicos y su impacto en el cuidador, $41 \%$ de los casos estaban institucionalizados, haciendo difícil una comparación de los resultados, ya que las características de las muestras son diferentes.

Al considerar las características del cuidador, sólo se observa que los cuidadores con sobrecarga intensa le dedican más tiempo al paciente. En este sentido, los esposos/as les dedican significativamente más tiempo que los hijos al paciente, no siendo el parentesco sino el tiempo lo que se correlaciona con la sobrecarga. Los otros factores como sexo, edad o nivel cultural, no se observaron como diferencias significativas respecto a la sobrecarga del cuidador. Este aspecto nos abre un posible camino de intervención en el sentido de tutelar una cantidad de tiempo libre recomendable para los cuidadores, que debería ser evaluado.

\section{Factores de la enfermedad que se relacionan a la sobrecarga}

El estadio motor evaluado por la UPDRS, la etapificación de Hoehn \& Yahr y los trastornos de conducta evaluados por el NPI mostraron una tendencia mayor en relación al aumento de la sobrecarga, en tanto la escala de actividades de Schwab and England y el MMP tendían a disminuir los puntajes. Ninguna de ellas alcanzó significación estadística. Sin embargo, resulta coherente en el sentido de que las personas con mayores trastornos motores, mayor deterioro cognitivo o trastornos conductuales provocan una mayor sobrecarga para el cuidador. Probablemente no alcance significación en la muestra, ya que está compuesta por pacientes con poco compromiso en estos ámbitos, demostrados claramente como factores de riesgo en estudios previos ${ }^{5,7}$.

Los trastornos de conducta se han demostrado como un factor que aumenta la sobrecarga del cuidador, especialmente en el grupo de paciente con demencia. La función memoria resultó ser la más afectada en el grupo de pacientes donde sus cuidadores presentaban una mayor sobrecarga, aunque esta tendencia no fue estadísticamente significativa. El IBD para depresión se muestra significativamente distinto, aumentando con la sobrecarga del cuidador en la ESZ, surgiendo un factor gravitante. Lo anterior había sido reportado previamente por otros autores ${ }^{6}$.

Un buen ánimo del paciente claramente contribuye a la calidad de vida de quienes lo rodean. En muchas ocasiones el diagnóstico del cuadro depresivo del paciente es difícil en el contexto de un paciente con una inhibición motora asociado a trastornos de ansiedad, por lo que con preguntas simples como la incluida en NPI sobre depresión, no se logran discriminar. En este sentido, una evaluación sistemática con el uso de escalas auto-aplicadas como el IBD puede ser de utilidad en la detección más objetiva, lo que junto con la educación del cuidador para que esté alerta a estos síntomas podría convertirse en una estrategia para enfrentar este problema. Evaluaciones clínicas globales no estructuradas pueden conducir al sobrediagnóstico y consecuente sobre-medicación de estos pacientes.

\section{Componentes de la escala de sobrecarga de Zarit}

El primer factor y el más preponderante en la escala es el impacto en el cuidador que hace referencia a aquellas cuestiones relacionadas con los efectos que la prestación de cuidados tiene sobre éste. Algunos de estos pueden ser la falta de tiempo libre, falta de intimidad, deterioro de la vida social, sensación de pérdida de control sobre su vida, entre otros. El segundo factor es el interpersonal, que representa aspectos de la relación que el cuidador mantiene con el paciente, como deseos de delegar el cuidado a otros, sentimientos de vergüenza, irritabilidad, tensión familiar etc. Y el último factor y menos representado en la escala, refleja creencias del cuidador sobre su capacidad para cuidar al paciente, como el deber hacerlo, los recursos económicos y otros.

El IBD se correlaciona con todos los factores 
ya debatidos. El factor de impacto se correlaciona mejor con los factores que reflejan incapacidad física, en tanto el factor interpersonal está mejor relacionado con aspectos cognitivos, en especial la alteración de la memoria. Esta observación es concordante con lo publicado por otros autores ${ }^{19}$.

\section{Como enfocar la educación y el cuidado de los cuidadores ${ }^{28,29}$}

La enfermedad de Parkinson es una enfermedad crónica y progresiva. Precisamente, su progresión determina un aumento de la carga del cuidador, que entendemos como el impacto subjetivo y objetivo que éste percibe sobre su salud, vida social y estado financiero, producto de su experiencia de cuidador ${ }^{19}$. El nivel de sobrecarga evidencia que los parientes no están típicamente familiarizados al asumir el rol de cuidador o el manejo emocional y físico que demandan los pacientes con EPI.

El 40\% de los pacientes con EPI desarrollan depresión. Nuestros datos muestran que existe una correlación estrecha entre los síntomas depresivos del paciente y el aumento de la carga del cuidador. Lo anterior se podría explicar por la percepción del paciente y de su cuidador del poco control sobre sus síntomas y de la no predictibilidad de la enfermedad. La depresión de los pacientes con EPI ha demostrado ser un gran factor determinante en la sobrecarga del cuidador, más que la severidad motora funcional y mental de los pacientes con EPI. Finalmente, el estrés del cuidador afecta no sólo a él mismo sino también al paciente, ya que es uno de los factores más importantes en el aumento de la incidencia de maltratos ${ }^{30}$. Frente a ello, es importante que el cuidador y paciente puedan contar con un soporte social que sería un poderoso mediador o buffer para la relación. El soporte social reduce el estrés, la carga percibida y promueve la salud del cuidador.

\section{Conclusiones}

Los resultados de este estudio nos muestran que los pacientes con EPI experimentan altos índices de depresión, lo que es un importante factor de riesgo para la sobrecarga de los cuidadores. Otros factores como el deterioro cognitivo y el compromiso motor, especialmente la marcha y el equilibrio, también sobrecargan al cuidador, lo que concuerda con los trabajos de otros autores ${ }^{3}$. El manejo del cuadro depresivo seria un factor relevante para mejorar la calidad de vida, tanto de los cuidadores como de los pacientes.

\section{Referencias}

1. Errea JM, Ara JR, Aibar C, de Pedro-Cuesta J. Prevalence of Parkinson's disease in lower Aragon, Spain. Mov Disord 1999; 14 (4): 596-604.

2. Chiófalo N, Kirschbaum AI, Schoenberg B, Olivares O, Valenzuela M, Soto E, Álvarez G. Estudio epidemiológico de las enfermedades neurológicas en Santiago Metropolitano, Chile/Epidemiological study of neurological diseases in Metropolitan Santiago, Chile. Rev Chil Neuro-Psiquiatr 1992; 30 (4): 335-40.

3. Shulman LM, Taback RL, Bean J, Weiner WJ. Comorbidity of the nonmotor symptoms of Parkinson's disease. Mov Disord 2001; 16 (3): 507-10.

4. Juri C, Viviani P, Chaná P. Features associated with the development of non-motor manifestations in Parkinson's disease. Arq Neuropsiquiatr 2008; 66 (1): 22-5.

5. Aarsland D, Larsen JP, Karlsen K, Lim NG, Tandberg E. Mental symptoms in Parkinson's disease are important contributors to caregiver distress. Int J Geriatr Psychiatry 1999; 14 (10): 866-74.

6. Fernández HH, Tabamo RE, David RR, Friedman JH. Predictors of depressive symptoms among spouse caregivers in Parkinson's disease. Mov Disord 2001; 16 (6): 1123-5.

7. Martínez-Martín P, Guerrero-Díaz MT, Frades-Payo B. [Neuropsychological disorders in Parkinson's disease: evaluating them and their impact on the caregiver]. Rev Neurol 2004; 39 (7): 639-45.

8. Edwards NE, Scheetz PS. Predictors of burden for caregivers of patients with Parkinson's disease. J Neurosci Nurs 2002; 34 (4): 184-90.

9. Regueiro Martínez AA, Pérez-Vázquez A, Gomara Villabona SM, Ferreiro Cruz MC. [Short Zarit interview on burden of care for caregivers in primary care]. Aten Primaria 2007; 39 (4): 185-8.

10. Rivas J, Ostiguin R. Cuidador: ¿concepto operativo o preludio teorico? Revista Enfermeria Universitaria ENEO-UNAM 2011; 8 (8): 49-54.

11. Chaná P, Miranda M, Villagra R, Salinas R, Cox P, Valdivia F. Diagnóstico, evaluación y tratamiento de la enfermedad de Parkinson: Guía Práctica. Rev Chil Neuro-Psiquiatr 2001; 39 [Supplemento 1]: 1-56.

12. The Unified Parkinson's Disease Rating Scale (UPDRS): 
Sobrecarga de cuidador en la enfermedad de Parkinson - O. Benavides et al

status and recommendations. Mov Disord 2003; 18 (7): 738-50.

13. Juri-Claverías C, Aguirre M, Viviani G, Chaná-Cuevas P. [Risk factors associated with the development of motor complications in Parkinson's disease. A study in a Chilean population]. Rev Neurol 2007; 45 (2): 77-80.

14. Parrao-Díaz T, Chaná-Cuevas P, Juri-Claverías C, Kunstmann C, Tapia-Núñez J. [Evaluation of cognitive impairment in a population of patients with Parkinson's disease by means of the mini mental Parkinson test]. Rev Neurol 2005; 40 (6): 339-44.

15. Boada M, Cejudo JC, Tarraga L, López OL, Kaufer D. [Neuropsychiatric inventory questionnaire (NPI-Q): Spanish validation of an abridged form of the Neuropsychiatric Inventory (NPI)]. Neurologia 2002; 17 (6): 317-23.

16. Vilalta-Franch J, Lozano-Gallego M, Hernández-Ferran$\operatorname{diz}$ M, Llinas-Regla J, López-Pousa S, López OL. [The Neuropsychiatric Inventory. Psychometric properties of its adaptation into Spanish]. Rev Neurol 1999; 29 (1): 15-9.

17. Leentjens AF, Verhey FR, Luijckx GJ, Troost J. The validity of the Beck Depression Inventory as a screening and diagnostic instrument for depression in patients with Parkinson's disease. Mov Disord 2000; 15 (6): 1221-4.

18. Zarit SH, Reever KE, Bach-Peterson J. Relatives of the impaired elderly: correlates of feelings of burden. Gerontologist 1980; 20 (6): 649-55.

19. Montorio I, Fernández M, López A, Sánchez M. La Entrevista de Carga del Cuidador. Utilidad y validez del concepto de carga. Anales de Psicología 1998; 14 (2): 229-48.

20. Breinbauer K, Vásquez V, Mayanz S, Guerra C, Millán K. Validación en Chile de la Escala de Sobrecarga del
Cuidador de Zarit en sus versiones original y abreviada. Rev Med Chile 2009; 137: 657-65.

21. Chaná P, Kunstmann C. Influencia de la edad en los síntomas motores de la enfermedad de Parkinson. Rev Chil Neuro-Psiquiatr 2004; 42 [Supl 1]: 11-12.

22. Chaná P, Galdames D. [Accessibility to the specific pharmacotherapy for Parkinson disease, in Santiago de Chile]. Rev Med Chile 1998; 126 (11): 1355-61.

23. Banco de instrumentos básicos para la práctica de la psiquiatría clínica. Segunda Edición ed. Barcelona: Ars Médica; 2003.

24. Errea JM, Ara JR. [Depression and Parkinson disease]. Rev Neurol 1999; 28 (7): 694-8.

25. Aarsland D, Andersen K, Larsen JP, Lolk A, KraghSorensen P. Prevalence and characteristics of dementia in Parkinson disease: an 8-year prospective study. Arch Neurol 2003; 60 (3): 387-92.

26. Errea JM, Ara JR. [Cognition deterioration in Parkinson's disease: associated risk factors]. Rev Neurol 1999; 28 (5): 439-43.

27. Zarit SH. Behavioral disturbances of dementia and caregiver issues. Int Psychogeriatr 1996; 8 Suppl 3: 263-8.

28. Edwards NE, Ruettiger KM. The influence of caregiver burden on patients' management of Parkinson's disease: implications for rehabilitation nursing. Rehabil Nurs 2002; 27 (5): 182-6, 198.

29. Secker DL, Brown RG. Cognitive behavioural therapy (CBT) for carers of patients with Parkinson's disease: a preliminary randomised controlled trial. J Neurol Neurosurg Psychiatry 2005; 76 (4): 491-7.

30. Sánchez-Guzmán MA, Paz-Rodríguez F. [Violence in the caregiver-patient dyad in Parkinson disease: 3 measurement methods]. Salud Pública Mex 2011; 53 (3): 203-4. 